\title{
Eletroconvulsoterapia como prática psiquiátrica: revisão de literatura
}

\author{
Electroconvulsive therapy as psychiatric practice: literature review
}

La electroconvulsoterapia como práctica psiquiátrica: revisión de la literatura

Bruno Braga José

Marlene Cabral Coimbra da $\mathbf{C R U Z}^{2}$

${ }^{1}$ Acadêmico do Curso de Medicina da Universidade Brasil - Campus Fernandópolis 15600-000 Fernandópolis-SP, Brasil

${ }^{2}$ Docente dos Cursos de Medicina e Odontologia da Universidade Brasil - Campus Fernandópolis, 15600-000 Fernandópolis-SP, Brasil

\section{Resumo}

Introdução: A eletroconvulsoterapia é um procedimento que utiliza uma corrente elétrica para induzir uma crise convulsiva sob anestesia geral, com o objetivo de produzir alterações no comportamento e melhoras nos sintomas psiquiátricos. Objetivo: revisar a literatura com relação ao procedimento, indicações, contraindicações e implicações éticas na prática psiquiátrica. Material e Método: foi realizada uma pesquisa de livros texto e de publicações obtidas nas bases de dados BVS, Medline e Scielo sobre o tema. Resultados: Apesar de todos os avanços, o mecanismo de ação da ECT ainda é desconhecido, e existem diversas teorias sobre seu funcionamento. É tratamento de primeira escolha nos casos de necessidade de melhora rápida, como depressões graves e grande risco de suicídio, e como segunda escolha, quando existe ausência de resposta terapêutica satisfatória de psicofármacos. É preciso avaliar os riscos cardiológicos decorrentes da anestesia, e testes neuropsicológicos são importantes para acompanhar alterações cognitivas que podem ocorrer. O Conselho Federal de Medicina reconhece a importância deste método terapêutico e regulamenta sua aplicação e cuidados, sendo um ato médico a ser realizado em ambiente com infraestrutura adequada de suporte à vida e a procedimentos anestésicos e de recuperação. Conclusão: a eletroconvulsoterapia é um tratamento eficaz para remissão de sintomas graves psiquiátricos, principalmente quando se precisa de resultados rápidos. Porém sua prática ainda é controversa, apesar de ser regulamentada pelo Conselho Federal de Medicina.

Descritores: Eletroconvulsoterapia; Eletricidade; Psiquiatria.

\section{Abstract}

Introduction: Electroconvulsive therapy is a procedure that uses an electric current to trigger a seizure under general anesthesia, with the aim of producing changes in behavior and better in psychiatric symptoms. Objective: To review the indication regarding the procedure, indications, contraindications and ethical policies in the psychiatric practice. Material and Method: a research of textbooks and information about the practice in Medline and Scielo databases. Results: Despite all the advances, the mechanism of action of ECT is still unknown, and there are several theories about its functioning. First choice in serious cases, such as severe depressions and great risk of suicide, and second choice in case of absence of therapeutic response to psychotropic drugs. Cardiological risks of anesthesia and neuropsychological tests are important to follow the cognitive changes that may occur. The Federal Medical Council approves and regulates its application and care, as being an physician-needed procedure in na environmentally oriented with adequate life support capacity and one of the anesthetic and recovery procedures. Conclusion: electroconvulsive therapy is an effective treatment for the remission of serious psychiatric errors, especially when fast results are needed. Its practice is still controversial, although it is regulated by the Federal Council of Medicine.

Descriptors: Electroconvulsive Therapy; Electricity; Psychiatry.

\section{Resumen}

Introducción: La electroconvulsoterapia es un procedimiento que utiliza una corriente eléctrica para desencadenar una crisis convulsiva bajo anestesia general, con el objetivo de producir alteraciones en el comportamiento y mejor en los síntomas psiquiátricos. Objetivo: revisar la indicación con relación al procedimiento, indicaciones, contraindicaciones y políticas éticas en la práctica psiquiátrica. Material y Método: se realizó una investigación de libros de texto e informaciones sobre bases de datos BVS, Medline y Scielo sobre el tema. Resultados: A pesar de todos los avances, el mecanismo de acción de la ECT todavía es desconocido, y hay varias teorías sobre su funcionamiento. Como es el caso de casos graves, como depresiones graves y gran riesgo de suicidio, como es el caso del etéreo, la ausencia de respuesta terapéutica a los psicofármacos. Se evidencian los riesgos cardiológicos de la anestesia, y las pruebas neuropsicológicas son importantes para acompañar las alteraciones cognitivas que pueden ocurrir. El Consejo Federal de Medicina es un órgano de cuidado médico y regulador de su aplicación y cuidados, siendo un médico orientado al ambiente con capacidad adecuada de soporte a la vida ya uno de los procedimientos anestésicos y de recuperación. Conclusión: la electroconvulsoterapia es un tratamiento eficaz para la remisión de graves errores psiquiátricos, principalmente cuando se necesitan resultados rápidos. Su práctica sigue siendo controvertida, a pesar de estar regulada por el Consejo Federal de Medicina. Descriptores: Terapia Electroconvulsiva; Electricidad; Psiquiatría.

\section{INTRODUÇÃO}

A eletroconvulsoterapia (ECT), também conhecida como eletrochoque, é um procedimento que utiliza uma corrente elétrica para induzir uma crise convulsiva sob anestesia geral. ${ }^{1}$ É uma estimulação cerebral não farmacológica com o objetivo de produzir alterações no comportamento e melhoras nos sintomas psiquiátricos. ${ }^{2,3}$ Trata-se de um procedimento muito utilizado na psiquiatria e sua prática é regulamentada pelo Conselho Federal de Medicina através da resolução CFM no 2057/2013, só podendo ser realizada sob anestesia. ${ }^{4}$

A ECT geralmente é mal interpretada pela falta de informação da população e dos profissionais de saúde. O tema apresenta conteúdos distorcidos pela mídia no início da luta antimanicomial, além de depoimentos de familiares e pacientes que foram vítimas da má indicação e aplicação desta técnica. A falta da divulgação dos avanços da ECT contribui, de certa forma, para o estigma sobre esse procedimento até os dias de hoje. ${ }^{5}$

O Objetivo deste estudo foi de revisar a literatura com relação ao procedimento e mecanismo de ação, indicações e contraindicações e implicações éticas do uso da ECT na prática psiquiátrica.

\section{MATERIAL E MÉTODO}

Trata-se de uma pesquisa bibliográfica de livros texto sobre o tema e de publicações obtidas nas bases de dados BVS, Medline e Scielo, utilizando os descritores eletroconvulsoterapia, reforma psiquiátrica, psiquiatria.

\section{RESULTADOS E DISCUSSÃO}

Devido à falta de tratamentos eficazes na psiquiatria no início de século $\mathrm{XX}$, cientistas começaram a criar métodos, baseado na observação de poucos casos. Wagner-Juaregg em 1927 foi o 
pioneiro nos tratamentos biológicos em psiquiatria, através da malarioterapia, que era uma indução de febre para a melhora de sintomas psiquiátricos. A insulinoterapia foi desenvolvida por Meduna em 1933 e a lobotomia realizada por Egas Moniz em $1935^{6}$.

A ECT começou a ser utilizado com Cerletti e Bini em 1938, cercada de muitas críticas. Posteriormente, nas décadas de 60 e 70 , teve seu declínio, pelo avanço dos neurolépticos e pela movimentação da luta antimanicomial, pelo uso indevido e punitivo da técnica ${ }^{3}$.

A prática só retomou com sua credibilidade na década de 80 , pelos benefícios dessa terapia e pela incorporação do uso de aparelhos modernos, que tem a capacidade de dosar a carga exata para cada tratamento e gerar parâmetros mais fisiológicos, como a possibilidade de controlar o comprimento de onda utilizada, o controle do tempo e a frequência do disparo da corrente elétrica ${ }^{2}$.

A ECT moderna, conhecida como "ECT modificada", se diferencia da técnica antiga por fazer o uso de anestesia constituída por um hipnótico (propofol ou etomidato), um relaxante muscular (succinilcolina) e um fármaco que evita os efeitos vagais do procedimento (atropina). Conta também com monitorização cardíaca, oximetria, controle da pressão arterial e eletroencefalograma (EEG) para o acompanhamento da crise convulsiva. ${ }^{2} \mathrm{O}$ Instituto de Psiquiatria da Universidade Federal do rio de Janeiro (IPUB-UFRJ) faz uso da ECT desde $1946^{6}$.

\section{- Mecanismo de ação}

Apesar de todos os avanços, o mecanismo de ação da ECT ainda é desconhecido, e existem diversas teorias sobre seu funcionamento. Na teoria das monoaminas, a corrente elétrica direcionada ao cérebro aumentaria a neurotransmissão adrenérgica, dopaminérgica e serotoninérgica. Já na teoria anticonvulsivante, o tempo de convulsão diminuiria e o limiar desta aumentaria. Outra teoria, a neuroendócrina, propõe que a relação com a melhora dos quadros psiquiátricos, seria pela liberação de hormônios como a prolactina, hormônio estimulador da tireoide e endorfinas. Por fim, na teoria neurotrófica, acredita-se que a ECT estimula a neurogênese e aumenta a sinalização no cérebro ${ }^{1}$.

Outra teoria que Saddock et al. $^{7}$ acrescentaram, coloca uma redução do metabolismo cerebral após a convulsão induzida por ECT com a melhora dos sintomas. Essa teoria foi elucidada após a utilização de tomografia por emissão de prótons, revelando que durante a convulsão houve um aumento do fluxo sanguíneo, o consumo de glicose e de oxigênio no cérebro, além do aumento da permeabilidade da barreira hematencefálica. Por outro lado, após a convulsão, o fluxo do sangue e o consumo da glicose foram reduzidos, sobretudo, de forma mais acentuada no lobo frontal.

\section{○ Indicações}

A ECT é a primeira escolha nos casos em que há necessidade de melhora rápida, como por exemplo pacientes com depressões graves e grande risco de suicídio. Como tratamento de segunda escolha, é indicado quando existe ausência de resposta terapêutica satisfatória de psicofármacos. ${ }^{3}$ Segundo Neto e Elkis ${ }^{8}$, a ECT é o tratamento de primeira escolha em portadores de transtorno depressivo grave, pela presença de sintomas psicóticos ou catatônicos, pelo risco de suicídio ou pela necessidade de rápida resposta; e segundo Forlenza ${ }^{9}$ também é primeira escolha para a Síndrome Neuroléptica Maligna.

Há concordância entre vários autores na indicação em casos de depressão monopolar, no transtorno bipolar e na esquizofrenia, mas divergência nos casos de psicoses; delirium tremens, doença de Parkinson, depressão pós-parto, entre outras ${ }^{3}$.

Já Madeira et al. ${ }^{10}$ destacam a importância da indicação da ECT em pacientes com psicose puerperal pois reduz o período sintomático, diminuindo os riscos de suicídio ou infanticídio e restabelecendo rapidamente a relação mãe-lactente possibilitando a amamentação materna.

Alguns autores colocaram a existência de boa resposta em casos de Doença de Parkinson e depressão pós-parto suicida ${ }^{11}$, sintomas de humor em pacientes com hidrocefalia ${ }^{12}$, e ainda em pacientes com doença de Huntington ${ }^{13}$.

Segundo estudo de Pastore et al. ${ }^{6}$, dos 69 pacientes tratados com ECT na IPUB-UFRJ de 2005 a 2007, as indicações foram, por ordem decrescente de uso, heteroagressividade, tentativa de suicídio, auto agressividade, refratariedade aos psicofármacos, esquizofrenia, depressão grave, mania grave, catatonia e por gravidez.

\section{- Contra-indicações}

Segundo Forlenza ${ }^{9}$ não existem contraindicações absolutas à ECT, mas relativas como um processo expansivo ou hipertensão intracraniana, e doenças ortopédicas, já que a ECT provoca contrações musculares em decorrência à convulsão induzida.

Há algumas situações clínicas que requerem cuidados específicos por implicarem maiores riscos: situações cardiovasculares graves (infarto recente, angina instável, doenças coronarianas, insuficiência cardíaca congestiva), aneurismas ou malformações vasculares, acidente vascular cerebral recente, algumas condições pulmonares, além de condições de risco anestésico ${ }^{14}$.

Deve-se também contra-indicar este procedimento quando o cliente tiver qualquer outra condição associada a um escore de risco anestésico 4 ou 5 da ASA, American Society of Anesthesiologists ${ }^{15}$. 
$\mathrm{O}$ uso de anticonvulsivantes e benzodiazepínicos impede o tratamento com ECT, enquanto o carbonato de lítio está associado a delirium prolongado. Esses agentes devem ser temporariamente interrompidos ou diminuídos antes da ECT. Já o uso da maioria dos antidepressivos é seguro durante a ECT, com exceção dos inibidores da monoamina oxidase (IMAO) ${ }^{11}$

\section{- Efeitos colaterais}

Embora aconteça a diminuição dos sintomas depressivos com a melhora de algumas funções neurocognitivas como a atenção, concentração, inteligência geral, raciocínio abstrato e criatividade, existem alguns efeitos adversos ${ }^{16}$, Os déficits cognitivos podem ser inexistentes, leves ou até graves, sendo sempre reversíveis ${ }^{9}$. O distúrbio cognitivo mais afetado é a memória, mas também podem ser afetadas a orientação e a linguagem ${ }^{9}$. Outros efeitos adversos são estados confusionais, a cefaleia e a náusea ${ }^{3}$. Também é possível que o paciente tenha episódios breves de hipotensão ou hipertensão arterial, bradi ou taquiarritmias ${ }^{17}$. Não existem evidências científicas de que a ECT possa gerar danos neuronais. Ao contrário, há ocorrência de neurogênese ${ }^{15}$.

A taxa de mortalidade associada à ECT é similar àquela associada aos procedimentos envolvendo anestesia geral, sendo que os eventos que resultaram em morte ocorreram quase que exclusivamente em função de complicações cardíacas $^{7}$. Segundo Alvarenga e Andrade ${ }^{15}$, a taxa de óbitos varia de 2 a $4,5 \%$ e a de complicações 1 a cada 1500 tratamentos.

\section{- Procedimento}

É necessário realizar uma avaliação clínica com anamnese médica completa e exame físico para excluir patologias e riscos, evitando ao máximo qualquer tipo de complicação relacionada com esta técnica?

Também é prudente a realização de um hemograma completo para afastar anemias, quadros infecciosos e inflamatórios; International Normalized Ratio (INR) e Partial Thromboplastin Time (PTT), indicados para pacientes que fazem o uso de anticoagulantes; a glicemia basal, uma vez que a ECT diminui o metabolismo da glicose; as provas de função renal (ureia e creatinina) e de função hepática (transaminase glutâmico-pirúvica, transaminase glutâmico-oxalacética e gama-glutamil transpeptidase) para nortear quais os medicamentos que serão utilizados durante o procedimento; hormônio estimulante da tireoide (TSH) deve ser dosado, já que a ECT gera o aumento oxcarbazepina para evitar convulsões frustadas e confusão mental. ${ }^{18}$

Exames complementares como radiografia de tórax e da espinha dorsal, eletroencefalograma (EEG), eletrocardiograma (ECG) e avaliação préanestésica são necessários ${ }^{3}$.
Deve-se também avaliar os riscos cardiológicos decorrente da anestesia, e testes neuropsicológicos devem ser realizados antes e depois da ECT, importantes para acompanhar diversas alterações cognitivas, principalmente a memória. ${ }^{18}$

O paciente precisa de um jejum de seis a oito horas antes da ECT e todas as próteses ou aparelhos removíveis devem ser removidos cavidade oral. ${ }^{7} \mathrm{Na}$ hora da sessão, é necessário proteger o paciente dos movimentos extremos da convulsão, que ocasionariam fraturas. Coloca-se um protetor na boca para se prevenir mordidas e deslocamentos de mandíbula. $^{3}$

Utiliza-se fármacos anticolinérgicos muscarínicos como drogas pré-anestésicas para reduzir secreções e impedir as bradiarritmias. ${ }^{17} \mathrm{~A}$ atropina é mais comumente utilizada pela via intramuscular (IM), subcutânea (SC) ou intravenosa (IV). Também pode-se utilizar glicopirrolato por via IM, SC ou IV. ${ }^{7}$ Entretanto, Rosa \& Rosa ${ }^{18}$ (2015), refere que não é necessário o uso de drogas préanestésicas justificando pela associação com o prolongamento do período de recuperação.

Os anestésicos de primeira escolha na ECT são o metoexital e o propofol. Ambos são eficazes por apresentarem a meia-vida curta e serem pouco cardiotóxicos. ${ }^{19}$ Outras alternativas são o etomidato, a cetamina e a alfetanila. ${ }^{7}$ Para Rosa et al..$^{20}$, o propofol tem o tempo de recuperação bem mais curto quando comparado ao etomidato e, segundo Associação Brasileira de Psiquiatria ${ }^{19}$, este é o agente com menor cardiotoxicidade. Jesus ${ }^{1}$ em estudo que avaliou anestésicos e eficácia da ECT na depressão maior, observou que os anestésicos mais utilizados foram o propofol e o tiopental; sendo que o propofol, apesar de apresentar menor tempo de convulsão, reduz o déficit cognitivo, tempo de recuperação e tempo de emergência, além de previnir a elevação da pressão arterial inerente ao ECT e seu uso é benéfico em pacientes com risco cardíaco.

A succinilcolina deve ser utilizada como relaxante muscular com a função de evitar fraturas ou lesões, já que impede a movimentação do paciente. ${ }^{7}$ Entretanto, essa droga está contraindicada em clientes suscetíveis à hipertermia maligna e a síndrome neuroléptica maligna ${ }^{18}$.

Segundo Antunes et al. $^{21}$, após a anestesia são colocados os eletrodos de forma unilateral (no hemisfério não dominante, geralmente o direito), bitemporal, bilateral ou bifrontal. Mas, os posicionamentos mais comumente utilizados são o bitemporal e o bilateral, pois proporcionam uma resposta mais eficaz e rápida, embora estejam associados à maior intensidade de déficits cognitivos ${ }^{9}$. Já na ECT unilateral direita, foi observado que existe menos efeitos colaterais que nas demais técnicas ${ }^{7}$. Atualmente, o posicionamento 
bifrontal vem ganhando relevância por apresentar eficácia e menores efeitos colaterais cognitivos ao comparado com o bitemporal e unilateral direito ${ }^{19}$.

Colocado os eletrodos no escalpe do sujeito, é administrado um estímulo elétrico forte o suficiente para gerar uma crise convulsiva ${ }^{7}$. Sua corrente elétrica é constante, com a possibilidade de modificar a voltagem, a frequência e o tamanho do pulso que pode ser breve (de 0,5 a 2,0 milissegundos) ou ultrabreves (menores que 0,5), cujo benefícios são semelhantes entre $\mathrm{si}^{19}$.

A dosagem da carga do estímulo elétrico é determinada a partir da titulação do limiar convulsígeno. Essa titulação é estipulada por meio de diversas administrações de estímulos elétricos com cargas crescentes até o momento em que a convulsão é obtida. Após ter o conhecimento do limiar convulsígeno do paciente, a dosagem depende do posicionamento dos eletrodos, ou seja, no posicionamento bitemporal e bifrontal a carga deverá equivaler a 1,5 a 2 vezes o limiar convulsígeno, enquanto o unilateral, seis vezes Entretanto, Bueno ${ }^{22}$ esclarece que podem ser aplicados no máximo quatro estímulos elétricos numa única sessão para este método de titulação.

Segundo Saddock et al. ${ }^{7}$ a convulsão em um paciente sob a ECT acontece pela despolarização de um grande número de neurônios simultaneamente. Esta despolarização altera o potencial elétrico extracelular que, por sua vez, gera a despolarização de neurônios vizinhos e propaga a atividade convulsiva por todo o cérebro. Após todo esse processo, as células utilizam de mecanismos para a homeostase para finalizar com a convulsão.

Bueno $^{22}$ detalha que logo após o estímulo elétrico, acontece uma resposta parassimpática intensa que é explicada pelo estímulo vagal, uma vez que o paciente executa uma manobra de Valsalva induzida por uma forte expiração associada com o fechamento da glote. Nesta fase, encontra-se uma bradicardia e a redução da pressão arterial. Ao final dessa descarga vagal, segue uma resposta simpática por consequência da generalização da crise convulsiva, resultando no aumento da frequência cardíaca e na pressão arterial. Ao final da convulsão, acontece uma resposta parassimpática, menos intensa que a inicial que é responsável por manter a frequência cardíaca e níveis pressóricos em níveis basais.

Neto e Elkis ${ }^{8}$ descreveram que, após o indivíduo acordar, acontece uma outra descarga do sistema simpático que pode ser potencializada pela agitação pós-ictal. E quando o paciente se torna totalmente vígil, acontece uma outra resposta parassimpática que retorna a frequência cardíaca e a pressão sanguínea ao seu nível basal.

Saddock et al. $^{7}$ relataram que durante a passagem da corrente elétrica, observam-se contrações musculares, principalmente da mandíbula e dos músculos faciais, mas que são independentes da convulsão. A convulsão é composta pela fase tônica (caracterizada pela extensão plantar) e pela fase clônica (caracterizada por diversas contrações rítmicas que vão diminuindo até cessar).

Para esta crise convulsiva ser eficaz, o tempo de duração não deverá ser menor que 15 segundos ${ }^{1}$. Para Neto e Elkis ${ }^{8}$, a monitorização do tempo da convulsão se faz pelo EEG associado ou não com a técnica de garroteamento de um membro por um manguito de esfigmomanômetro. Porém, esta técnica não é obrigatória já que a dose do relaxante muscular é baixa e apenas ameniza os movimentos e não os elimina em sua totalidade.

São descritos quatro tipos de crises indesejadas. A primeira é a crise frustra que não é suficiente para a generalização da crise e tem risco de bradiarritmia já que só ocorre a estimulação parassimpática. A segunda é a crise inadequada, pelo qual ocorre a generalização da crise, entretanto sua duração é inferior a 15 segundos, cuja eficácia é inferior ao desejado. A terceira é a crise prolongada cujo tempo ultrapassa dois minutos e tem risco de lesão cerebral por anoxia prolongada. Esta crise deve ser abortada com Diazepam ou Midazolam. A última é a crise reentrante sendo caracterizada por um início de nova crise sem outro estímulo elétrico e a conduta é a mesma da crise prolongada ${ }^{8}$.

A ECT é composta pela fase inicial e pela fase de manutenção. Na primeira, é programado de seis a doze sessões de ECT de duas a quatro vezes pela semana. A segunda é indicada para clientes que tiveram reexacerbações dos sintomas após a fase inicial ou quando a farmacoterapia não é tolerada e suas sessões podem ser realizadas semanalmente, quinzenalmente ou mensalmente. Entretanto, a duração do tratamento depende da gravidade dos sintomas, da disponibilidade do serviço e dos efeitos colaterais ${ }^{9}$.

O tratamento de manutenção à longo prazo consiste em uma abordagem profilática para tentar evitar recidivas futuras. A duração desta última fase depende da frequência de episódios depressivos prévios e da gravidade da doença ${ }^{23}$.

A manutenção pode ser realizada com a própria ECT, principalmente em pacientes que tenham respondido inteiramente pela fase inicial e que não foram eleitos para a manutenção farmacológica. $^{24,23}$ Salleh et al. ${ }^{23}$, em 2006, acrescentaram que é fortemente recomendado o uso de antidepressivos durante 16 a 20 semanas após a remissão dos sintomas pela ECT em pacientes com depressão. Todavia, para pacientes diagnosticados com esquizofrenia, foi observado que a continuação da ECT associado aos antipsicóticos é mais eficaz que o uso de antipsicóticos sozinhos ${ }^{25}$.

A ECT é responsável pela melhora dos 
sintomas e da qualidade de vida em pacientes com transtornos psiquiátricos, e que segundo Andreasen e Black $^{17}$ a taxa de melhora em pacientes deprimidos é de 70 a 80\%. Em relação à catatonia, estes autores relatam ser comum resposta em menos de cinco aplicações e, não raro, em apenas uma. Por outro lado, para mania aguda espera-se um número médio de oito a 12 aplicações e 16 nos casos difíceis ${ }^{23}$, e uma resposta quase que imediata com redução ou resolução do comportamento suicida ${ }^{11}$.

\section{- Opinião de pacientes e psiquiatras}

Em pesquisa realizada por Campos e $\mathrm{Higa}^{26}$ com 37 pacientes internados (100\%) em Unidade de Psiquiatria do Hospital de Clínicas da UNICAMP, independente de terem sido ou não submetidos à ECT anteriormente, $67,5 \%$ responderam serem favoráveis ao uso da ECT em si próprios e $32,44 \%$ opinaram negativamente ao seu uso. Sobre os familiares desse pacientes internados quando opinaram sobre sua autorização no uso do tratamento de ECT em seu familiar internado, $68,43 \%$ responderam afirmativamente, contra $31,57 \%$ ) que responderam negativamente.

Em entrevista a pesquisadora Tassis $^{27}$ um Psiquiatra contrário à ECT, relatou que em mais de 30 anos de profissão, recomendou a ECT a um único paciente, e esse paciente melhorou bem, mas que atualmente se questiona se teria realmente indicação, pois os novos medicamentos são muito mais efetivos e mais bem tolerados pelo organismo e com menos efeitos colaterais, e que se diz contrário ao uso rotineiro e como primeira indicação de tratamento. Já em entrevista com um Psiquiatra a favor da ECT, que indica e faz uso do procedimento, este afirmou que não deveria ser utilizada como primeiro tratamento, primeiramente é preciso passar por um ou dois antidepressivos, a não ser para depressão grave com risco de suicídio.

\section{- Implicações Éticas}

Segundo pesquisa realizada por Machado et al. $^{28}$, os aspectos éticos do uso da ECT não foram claramente tratados pelos estudos. Os enfoques se detiveram nas indicações, uso de equipamentos mais modernos, critérios de sedação e termo de consentimento da família.

A Resolução do Conselho Federal de Medicina $\mathrm{n}^{\circ} 2.057 / 2013^{4}$ reconhece a importância deste método terapêutico e regulamenta sua aplicação e cuidados, sendo um ato médico a ser realizado em ambiente com infraestrutura adequada de suporte à vida e a procedimentos anestésicos e de recuperação. Tem indicações específicas e não se trata de terapêutica de exceção, sendo obrigatória a avaliação do estado clínico geral do paciente antes da ECT, em especial as condições cardiovasculares, respiratórias e neurológicas. Só pode ser realizada com anestesia, e com máquinas modernas, registradas e certificadas pela Anvisa ${ }^{4}$.
A American Psychiatric Association (APA) e a Associação Brasileira de Psiquiatria estão envolvidas com os critérios de indicação, cabendo às instituições cumprirem as regras. A APA preconiza a ECT como tratamento de primeira escolha nos casos de necessidade de remissão rápida dos sintomas agudos, em que os riscos de outros tratamentos são maiores do que os da $\mathrm{ECT}^{6}$.

Para a realização da ECT, deve-se obter o consentimento após o esclarecimento de todo o procedimento pelo paciente, caso ele seja capaz de entender o propósito, os riscos e os benefícios do tratamento. Caso não seja ou não esteja capaz de consentir, o consentimento deve ser aprovado pela família ${ }^{18}$.

\section{CONCLUSÃO}

A eletroconvulsoterapia (ECT) é um tratamento eficaz para remissão de sintomas graves psiquiátricos, principalmente quando se precisa de resultados rápidos. Porém sua prática ainda é controversa, apesar de ser regulamentada pelo Conselho Federal de Medicina.

\section{REFERÊNCIAS}

1. Jesus GN. Anestésicos e eficácia da eletroconvulsoterapia em pacientes com depressão maior: revisão sistemática. Monografia do Curso de Medicina. Salvador: Universidade Federal da Bahia; 2015.

2. Shiozawa P, Netto GTM, Cordeiro Q, Ribeiro RB. Eletroconvulsoterapia para o tratamento de depressão psicótica refratária em paciente com desnutrição grave: estamos esquecendo a ECT? Rev Debates Psiquiatr. 2014;6-10.

3. Silva MLB, Caldas MT. Revisitando a técnica de eletroconvulsoterapia no contexto da reforma psiquiátria brasileira. Psicol cienc. 2008;28(2):344-61.

4. CFM, Conselho Federal de Medicina. Resolução $\mathrm{n}^{\circ}$ 2.057. Consolida as diversas resoluções da área da Psiquiatria e reitera os princípios universais de proteção ao ser humano, à defesa do ato médico privativo de psiquiatras e aos critérios mínimos de segurança para os estabelecimentos hospitalares ou de assistência psiquiátrica de quaisquer naturezas. Brasília: Diário Oficial da República Federativa do Brasil; 2013.

5. Ávila MD. Eletroconvulsoterapia: da origem à aplicação modificada [monografia]. Porto Alegre: Universidade Federal do Rio Grande do Sul; 2010.

6. Pastore DL, Bruno LM, Nardi AE, Dias AG. O uso da eletroconvulsoterapia no Instituto de Psiquiatria da Universidade Federal do Rio de Janeiro no período de 2005 a 2007. Rev Psiquiatr Rio Gd Sul. 2008;30(3):175-82.

7. Sadock BJ, Sadock VA, Ruiz P. Compêndio de psiquiatria. ciência do comportamento e 
psiquiatria clínica. 11. ed. Porto Alegre: Artmed; 2017.

8. Louzã MR Neto, Elkis HE. Psiquiatria Básica. 2.ed. Porto Alegre: Artmed; 2007.

9. Forlenza OV, Migues EC. Compêndio de clínica psiquiátrica. Barueri: Manole; 2012

10. Madeira N, Santos T, Relvas JS, Abreu JLP, Oliveira CV. Eletroconvulsoterapia no tratamento da psicose puerperal. J bras psiquiatr. 2012; 61(1):45-8.

11.Hales RE, Yudofsky SC, Gabbard GO. Tratado de Psiquiatria Clínica 5. ed. Porto Alegre: Artmed; 2012.

12.Barbosa IG, Rocha FL. Eletroconvulsoterapia na terapêutica da mania em paciente com hidrocefalia. J bras psiquiatr. 2008;57(4):276-79.

13.Yudofsky CS, Hales ER. Fundamentos de neuropsiquiatria e ciências do comportamento. 2. ed. Porto Alegre: Artmed; 2013.

14.Del Porto J. Atualidades sobre eletroconvulsoterapia. SNC em foco. 2006;2(2):16-26.

15. Alvarenga PG, Andrade AG. Fundamentos em psiquiatria. Barueri: Manole; 2008.

16. Antunes PB. Efeito da eletroconvulsoterapia sobre sintomatologia psiquiatria e qualidade de vida [dissertação]. Porto Alegre: Universidade Federal do Rio Grande do Sul - UFRS; 2008.

17. Andreasen CN, Black WD. Introdução a Psiquiatria. 2. ed. Porto Alegre: Artmed; 2011.

18. Rosa MA, Rosa MO. Fundamentos da eletroconvulsoterapia. Porto Alegre: Artmed; 2015.

19. Associação Brasileira de Psiquiatria (ABP). Eletroconsulsoterapia. Diretrizes AMB. 97p.

20. Rosa MA, Rosa MO, Belegarde IMT, Bueno CR, Fregni F. Recuperação pós-eletroconvulsoterapia: comparação entre propofol, etomidato e tiopental. Rev Bras Psiquiatr. 2008;30(2):149-51.

21. Antunes PB, Rosa MA, Belmonte-de-Abreu PS, Lobato MIR, Fleck MP. Eletroconvulsoterapia na depressão maior: aspectos atuais. Rev Bras Psiquiatr. 2009;31(supl 1):S26-33.

22.Bueno CR. Titulação do limiar convulsígeno e segurança cardiovascular[mestrado] São Paulo: Faculdade de Medicina da Universidade de São Paulo - USP; 2009.

23. Salleh MA, Papakostas I, Zervas I, Christodoulou G. Eletroconvulsoterapia: critérios e recomendações da Associação Mundial de Psiquiatria. Rev Psiq Clin. 2006;33(5):262-67.

24.Bauer M, Whybrow PC, Angst J, Versiani M, Möller HJ. Força-Tarefa da WFSBP sobre Diretrizes de Tratamento para Transtornos Depressivos Unipolares. Diretrizes da World Federation of Societies of Biological Psychiatry (WFSBP) para tratamento biológico de transtornos depressivos unipolares, $2^{\mathrm{a}}$ parte: tratamento de manutenção do transtorno depressivo maior e tratamento dos transtornos depressivos crônicos e das depressões subliminares. Rev Psiquiatr Clín. 2009; 36(supl 2):58-76.

25.Gul IG, Eryilmaz G, Sayar GH, Ozten E, Arat MM, Tarhan N. Avaliação da eficácia da eletroconvulsoterapia contínua para esquizofrenia resistente ao tratamento. Rev Psiq Clín. 2014; 41(4):90-4.

26.Campos CJG, Higa CMH. Opinião e conhecimento de pacientes e familiares sobre o uso da eletroconvulsoterapia: implicações para a enfermagem. Rev Esc Enferm USP. 1997;31(2):191-205.

27.Tassis FP. Cura, Castigo? Um estudo sobre a criação da eletroconvulsoterapia (ECT) e sua utilização em pacientes no estado do Espírito Santo [dissertação]. Universidade Federal do Espírito Santo - UFES; 2013.

28. Machado FB, Moraes-Filho IM, Fidelis A, Almeida RJ, Nascimento MSSP, Carneiro KC. Eletroconvulsoterapia: implicações éticas e legais. Rev Cient Sena Aires. 2018;7(3):235-47.

\section{CONFLITO DE INTERESSES}

Os autores declaram não haver conflitos de interesse.

\section{AUTOR PARA CORRESPONDENCIA}

\section{Marlene Cabral Coimbra da Cruz}

mcoimbracruz@gmail.com

Submetido em 23/10/2018

Aceito em 12/03/2019 